\title{
An open study of the effectiveness of a multi-component weight-loss intervention for adults with intellectual disabilities and obesity
}

\author{
Craig A. Melville ${ }^{1 *}$, Susan Boyle ${ }^{2}$, Susan Miller ${ }^{3}$, Susan Macmillan ${ }^{1}$, Victoria Penpraze ${ }^{4}$, Carol Pert ${ }^{3}$, \\ Dimitrios Spanos ${ }^{1}$, Lynsay Matthews ${ }^{1}$, Nicola Robinson ${ }^{3}$, Heather Murray ${ }^{5}$ and Catherine R. Hankey ${ }^{1}$ \\ ${ }^{1}$ Centre for Population and Health Sciences, College of Medical and Veterinary Life Sciences, Academic Centre, Gartnavel \\ Royal Hospital, University of Glasgow, 1055 Great Western Road, Glasgow G12 OXH, UK \\ ${ }^{2}$ Glasgow and Clyde Weight Management Service, NHS Greater Glasgow and Clyde, Glasgow, UK \\ ${ }^{3}$ Mental Health Partnership, NHS Greater Glasgow and Clyde, Glasgow, UK \\ ${ }^{4}$ Institute of Diet, Exercise and Lifestyle, Centre for Population and Health Sciences, University of Glasgow, Glasgow, UK \\ ${ }^{5}$ Robertson Centre for Biostatistics, Centre for Population and Health Sciences, University of Glasgow, Glasgow, UK
}

(Received 30 June 2010 - Revised 23 November 2010 - Accepted 24 November 2010 - First published online 24 January 2011)

\section{Abstract}

Adults with intellectual disabilities experience high rates of obesity. Despite this higher risk, there is little evidence on the effectiveness of weight-loss interventions for adults with intellectual disabilities and obesity. The present study examined the effectiveness of the TAKE 5 multi-component weight-loss intervention. Adults with obesity were invited using specialist intellectual disability services to participate in the study. Obesity was defined as a BMI of $30 \mathrm{~kg} / \mathrm{m}^{2}$ or greater. TAKE 5 included a daily energy-deficit diet of $2510 \mathrm{~kJ}(600 \mathrm{kcal})$, achieved via a personalised dietary prescription. Participants' body weight, BMI, waist circumference and levels of physical activity and sedentary behaviour were measured before and after the intervention. A total of fifty-four individuals consented to participate, of which forty-seven ( $87 \%)$ completed the intervention in the study period. There was a significant decrease in body weight (mean difference -4.47 ( $95 \%$ CI $-5.91,-3.03) \mathrm{kg} ; P<0.0001)$, BMI $\left(-1.82(95 \%\right.$ CI $\left.-2.36,-1.29) \mathrm{kg} / \mathrm{m}^{2} ; P<0.0001\right)$, waist circumference $(-6.29(95 \%$ CI -7.85 , $-4.73) \mathrm{cm} ; P<0.0001)$ and daily sedentary behaviour of participants $(-41 \cdot 40(95 \% \mathrm{CI}-62 \cdot 45,-20 \cdot 35) \mathrm{min} ; P=0.00034)$. Of the participants who completed the intervention, seventeen (36.2\%) lost $5 \%$ or more of their initial body weight. Findings from the study suggest that TAKE 5 is an effective weight-loss intervention for adults with intellectual disabilities and obesity. The effectiveness of TAKE 5 should be examined further in a controlled study.

Key words: Obesity: Weight loss: Intellectual disabilities: TAKE 5

Obesity is recognised as a major public health concern internationally, and the prevention and management of obesity is a priority for health care ${ }^{(1)}$. There is clear evidence of the negative impact of obesity on health ${ }^{(2)}$, with an increased risk of chronic health problems ${ }^{(3)}$ and increased mortality ${ }^{(4)}$.

A moderate, sustainable weight loss of $5-10 \%$ of initial body weight has been shown to be associated with significant clinical benefits in individuals with obesity ${ }^{(5)}$. The evidence used in clinical guidelines ${ }^{(6-8)}$ advocates the effectiveness of multi-component weight-loss interventions to support individuals to achieve clinically relevant weight loss. The National Institute for Health and Clinical Excellence and the Scottish Intercollegiate Guideline $\operatorname{Network}^{(7,8)}$ recommend that multi-component weight-loss interventions should include:
(1) dietary changes to create an energy-deficit diet of $2510 \mathrm{~kJ} / \mathrm{d}(600 \mathrm{kcal} / \mathrm{d})$,

(2) support to increase the levels of physical activity,

(3) the incorporation of behavioural methods to support sustained behavioural change.

The prevalence of obesity in adults with intellectual disabilities has been consistently reported to be higher in population-based studies than in general population studies $^{(9-14)}$. However, few studies have examined the effectiveness of weight-loss interventions for adults with intellectual disabilities and obesity ${ }^{(15)}$. None of the published studies has included an energy-deficit diet or met the current recommendations from clinical guidelines on the use of multi-component interventions for weight loss. Another

Abbreviations: GCWMS, Glasgow and Clyde Weight Management Service; IPAQ-S, International Physical Activity Questionnaire - short version; PAL, physical activity level; PDP, personalised dietary prescription.

*Corresponding author: Dr C. A. Melville, fax +44 141357 4899; email craig.melville@glasgow.ac.uk 
limitation of the evidence base is that no study has reported whether participants achieve clinically relevant weight-loss targets associated with clinical benefits $(5-10 \%$ of initial body-weight loss) ${ }^{(15)}$.

The aim of the present study was to examine the effectiveness and acceptability of a multi-component weight-loss intervention, incorporating an energy-deficit diet, for adults with intellectual disabilities and obesity. Specific research questions examined were whether participants achieve:

(1) a significant decrease in body weight,

(2) a mean weight loss of $5 \%$ of their initial body weight,

(3) a significant increase in the levels of physical activity,

(4) a reduction in time spent in sedentary behaviour.

\section{Methods}

The present study was conducted according to the guidelines laid down in the Declaration of Helsinki. In keeping with the Adults with Incapacity (Scotland) Act $2000^{(16)}$, all procedures involving human subjects/patients were approved by the Scotland Research Ethics Committee A and the relevant local research ethics committee. A detailed protocol of consent, in keeping with the Adults with Incapacity Act 2000, was implemented. This included seeking consent from individuals with intellectual disabilities with the capacity to provide informed consent, and seeking consent from the next of kin or welfare guardian in circumstances where the individual was unable to provide informed consent. Written informed consent was obtained from all participants or next of kin/ welfare guardian.

\section{Sample}

Potential participants in the present study were service users referred to intellectual disability dietitians, identified as having obesity and requesting support with weight loss. Also, these participants had been referred to intellectual disability dietitians by general practitioners (family physicians) in primary care or by other specialist intellectual disability professionals.

A total of 101 potential participants were identified from the dietitians' waiting list as meeting the inclusion criteria: over 18 years old; a BMI $\geq 30 \mathrm{~kg} / \mathrm{m}^{2}$; ambulatory. Since a specific approach to weight loss is recommended for persons with the Prader-Willi syndrome, they were excluded from the study. All 101 potential participants were invited to take part, of which fifty-four individuals who met the criteria for inclusion and provided informed consent participated in the study.

\section{Intervention}

TAKE 5 is based on the weight-loss intervention programme used in the Glasgow and Clyde Weight Management Service (GCWMS), of NHS Greater Glasgow and Clyde. It was adapted specifically for use with adults with intellectual disabilities, by a multidisciplinary group of clinical academics and health professionals. Whereas the GCWMS intervention is delivered via groups, TAKE 5 is an individual intervention involving family or paid carers to support participants, where appropriate. An individual intervention was believed to be more likely to be accessible to all adults with intellectual disabilities, who have diverse cognitive and communication abilities. Carers can support individuals to develop knowledge and skills relevant to weight loss and can provide encouragement and motivation for behavioural change. Previous studies have shown that the involvement of family and paid carers contributes to the effectiveness of weight-loss interventions for adults with intellectual disabilities ${ }^{(17,18)}$. To maximise the accessibility of the intervention and carer involvement, the sessions took place in each individual's home environment.

The intervention was delivered by two health professionals who had the experience of working with individuals with intellectual disabilities - a dietitian and a medically qualified, sports medicine graduate. Of the two health professionals, one worked with the first thirty-five participants (D. S.) and another with the subsequent nineteen participants (L. M.). Both received training and ongoing supervision at the GCWMS from professionals involved in the design of relevant aspects of the multi-component intervention.

TAKE 5 comprised nine sessions, designed to take place every 2-3 weeks. Each session lasted 40-60 min and followed a structured format, with specific defined content to be covered for each session. Completion of the intervention was defined as participation in all nine sessions. Accessible resources on the potential benefits of losing weight, eating a healthy balanced diet - based on the 'Eatwell' plate ${ }^{(19)}$ - and physical activity were developed for use by adults with intellectual disabilities and their carers.

The GCWMS and TAKE 5 interventions fully incorporate the recommendations of the National Institute for Health and Clinical Excellence guidelines for multi-component weightloss interventions for adults with obesity ${ }^{(7)}$.

Recommended dietary change was based on an individualised personalised dietary prescription (PDP) calculated to achieve an energy-deficit diet of $2510 \mathrm{~kJ} / \mathrm{d}(600 \mathrm{kcal} / \mathrm{d})$ and a weight loss of $0 \cdot 5-1 \mathrm{~kg} /$ week. BMR was calculated from sex, age and weight using the Schofield equation ${ }^{(20)}$. Total energy expenditure was calculated from BMR multiplied by a physical activity level (PAL) of $1 \cdot 4$. The daily energy intake used in the PDP was total energy expenditure minus $2510 \mathrm{~kJ}$ (600 kcal).

The PDP is based on five food groups that make up the Eatwell plate: starchy foods such as bread, rice, potatoes and pasta; meat/fish and alternatives; fruit and vegetables; milk and dairy products; foods high in sugar and fat ${ }^{(19)}$. The PDP also gave specific advice on portion sizes. Daily energy intake was limited to a minimum of $6276 \mathrm{~kJ}(1500 \mathrm{kcal})$ and was made up of a specified number of daily portions from these five food groups, including a daily allowance for treats. The individualised PDP aimed to ensure that $50 \%$ of daily energy intake was from carbohydrates (with contributions from starchy foods, fruit and vegetables, and dairy products), less than $35 \%$ from fats and less than $20 \%$ from protein, the five food groups and necessary micronutrients ${ }^{(21)}$. 
The current guidelines recommend that adults should aim to do a minimum of $30 \mathrm{~min}$ of accumulated, moderate-intensity physical activity, in bouts of not less than $10 \mathrm{~min}$, on at least $5 \mathrm{~d} /$ week $^{(22)}$. These were used as the basis for discussing individuals' current levels of physical activity and supporting participants to progressively increase this. Based on expressed preferences for physical activity, goals for achieving recommended intensity levels and increasing the time spent physically active were discussed. Current activity across three types of physical activity was reviewed with each participant and carer:

(1) Activity in the home environment as a replacement for sedentary behaviour, e.g. housework and gardening. Individuals were encouraged to increase their participation in activities in the home environment, in keeping with their level of abilities.

(2) Walking pedometers were given to each participant, and training was given on how to use these to measure daily steps taken. Based on the baseline average steps/d, individuals were encouraged to progressively increase walking. Participants were advised to wear the pedometers each day. Carers were asked to support the participants to make a note of steps taken. Walking targets were agreed in the TAKE 5 sessions.

(3) Sport and exercise information was given to each participant and carer on local leisure facilities and clubs with accessible sports and exercise groups/classes.

Behavioural methods should be incorporated into weight management programmes ${ }^{(7,8)}$ and are incorporated into each session of the TAKE 5 intervention. There are a range of appropriate strategies, and in line with the evidence, goal setting and self-monitoring are central to the TAKE 5 weight-loss intervention. Additional behavioural methods included within the TAKE 5 intervention include methods to maintain participant and carer motivation, cue avoidance, stimulus control and problem solving ${ }^{(7)}$.

Goal setting was negotiated with each participant, and their carers, on an individual basis. Where possible, participants identified relevant goals which were agreed after discussion with the carer and health professional. At the start of the intervention, the overall goal of a weight loss of $5 \%$ of initial body weight was discussed. At each session, one goal for dietary change and one for physical activity were discussed and agreed with participants and carers. The health professionals encouraged the participants to set realistic, achievable goals, and the aim for the participant was to meet these goals by the time of the subsequent session. Hence, at the start of each session, the health professional reviewed whether the participant had achieved the goals set at the previous session, discussing reasons for successful goal achievement and barriers to change where appropriate.

To allow participants and carers to monitor weight-loss success, participants were weighed at each session. Participants were invited to complete accessible paper diary sheets, with support from carers, to self-monitor physical activity and dietary changes between sessions. These optional diaries were reviewed and discussed in each session of the
TAKE 5 intervention. For some participants, it was not possible to complete the diaries due to the individual's level of intellectual disabilities or the support arrangements from carers. However, where possible, this information was used as part of the intervention, although the data were not used for the purposes of analysis.

\section{Measures}

At baseline and on completion of the TAKE 5 intervention, a research assistant who was not involved in delivering the intervention met with the participant and carers to complete the measures of outcome. Since the TAKE 5 sessions took place every 2-3 weeks, the follow-up period was approximately 24 weeks. Where possible, the researcher made every effort to involve the same carer at baseline and follow-up.

A purpose-designed data collection form was used to collect demographic and known health data on participants. Details of current medication prescribed were recorded. A systematic review of medications shown to be associated with significant weight gain was used to classify medications as obesogenic $^{(23)}$

The level of intellectual disabilities was categorised as mild, moderate, severe or profound. This was assessed with questions assessing an individual's level of ability and need for support in key areas of functioning. A total score (range $5-15$ ) is calculated by adding together the scores from the five individual questions. Cut-offs corresponding to the four categories of intellectual disabilities were derived in a previous study ${ }^{(24)}$ and shown to have a good level of agreement with a validated structured assessment of functioning and ability level, the Vineland's Adaptive Behaviour Scale ${ }^{(25)}$.

Measurements were made with the participant wearing light clothes without shoes. All measurements were made in duplicate. The final value was calculated as the mean of the two measurements. Weight $(\mathrm{kg})$ was measured to the nearest $100 \mathrm{~g}$, using Seca 877 scales (CE approval class III; Seca, Hamburg, Germany). Height (m) was measured to the nearest $1 \mathrm{~mm}$ using the Seca Leicester stadiometer (Seca). Height (m) and weight $(\mathrm{kg})$ were used to calculate BMI using the formula: $\mathrm{BMI}=$ weight $/$ height $^{2}\left(\mathrm{~kg} / \mathrm{m}^{2}\right)^{(26)}$. Waist circumference was measured to the nearest $0.5 \mathrm{~cm}$ at the midpoint between the iliac crest and the lowest rib, in full expiration with the participant standing ${ }^{(27)}$.

To objectively measure physical activity, participants were invited to wear accelerometers for $7 \mathrm{~d}$ before the start of the intervention, and at the end of the intervention period. Actigraph GT1M accelerometers (Manufacturing Technology, Inc., Fort Walton Beach, FL, USA) were worn at the hip, attached to a belt worn round the waist. Instructions were given to wear the Actigraph during all waking hours, except when showering, bathing or swimming. In keeping with the guidelines on the validity of accelerometer data, the minimum data requirement was set at $6 \mathrm{~h}$ of data on at least $3 \mathrm{~d}$ from 7 , to ensure a valid measure of $\mathrm{PAL}^{(28)}$. If this requirement was not met, the accelerometer data were not included in the analysis. Participants completed a diary to indicate wear times so that accurate activity levels could be calculated. 
The accelerometers were set to record activity over $15 \mathrm{~s}$ intervals (epochs). Activity counts of four consecutive epochs were summed to give activity counts/min. Published cut-offs were used to express the accelerometer data as three categories of activity intensity ${ }^{(29)}$ :

(1) sedentary behaviour 0-499 counts/min,

(2) light-intensity activity 500-1951 counts/min,

(3) moderate-to-vigorous-intensity activity >1952 counts/ $\min$.

The accelerometer data for each of the three categories of activity were expressed as mean time/d in min and percentage of total monitoring time.

Additional data on PAL were collected using the International Physical Activity Questionnaire - short version (IPAQ-S). The IPAQ-S involved cognitive demands and a level of abstraction that would be beyond the level of functioning of most participants. Therefore, it was completed by carers and participants together. The IPAQ-S has been shown to be a reliable and valid measure of physical activity $^{(30)}$ but has not been used previously in studies involving adults with intellectual disabilities.

\section{Statistical analysis}

Data were analysed using the statistical package SAS for Windows (version 9.1). Primary outcome change in weight at 24 weeks from baseline was analysed using a paired $t$ test. The results are reported as means and standard deviations for weight at baseline and 24 weeks along with the mean difference $(95 \% \mathrm{CI})$ and corresponding $P$ value. Continuous secondary outcomes (such as change in BMI) were analysed and reported in a similar manner. Physical activity measures were also analysed using a Wilcoxon signed-rank test, as the distribution of some measures was skewed. Categorical secondary outcome measures (e.g. eating five or more proportions of fruit and vegetables/d) were analysed using the exact McNemar test comparing responses at baseline with those at 24 weeks. The results are reported as the number and percentage of 'Yes' responses at baseline and 24 weeks along with the mean difference in percentages (95\% CI) and $P$ value from the exact McNemar test. Associations between patient characteristics and a weight loss of $5 \%$ or more of initial body weight were examined using logistic regression models. Weight changes at 24 weeks from baseline were also compared by patient characteristics (e.g. males $v$. females) using two-sample $t$ tests. Mean changes for each group are provided along with the mean difference (95\% CI) and associated $P$ values.

\section{Results}

A total of fifty-four individuals met the criteria for inclusion and consented to participate in the pilot study. Of the individuals, three dropped out, and four did not complete the intervention within the study period. Therefore, forty-seven (87.0\%) participants completed the intervention.

\section{Participant characteristics}

Baseline demographic and health characteristics of the fifty-four participants are shown in Table 1 . The mean age of participants was 48.3 (range 23-71, sD 12.01) years.

A significant proportion of participants had additional health needs and health problems commonly associated with obesity. Of the participants, nineteen $(35 \cdot 2 \%)$ reported difficulties in walking due to obesity and twelve (22.2\%) were prescribed obesogenic medication.

Due to ethical requirements of the present study, no data are available for individuals who chose not to take part. Compared with individuals with intellectual disabilities and obesity described in a population-based epidemiological sample, the participants in the present study sample were less able $\mathrm{e}^{(12)}$. However, the sex, age and prevalence of additional health problems were similar.

\section{Weight change and other anthropometric measures at 24 weeks}

There was a significant post-intervention decrease in weight (mean difference -4.47 (SD $4.75,95 \% \mathrm{CI}-5.91,-3.03) \mathrm{kg}$; $P<0.0001$ ), BMI (mean difference -1.82 (95\% CI $-2 \cdot 36$, $\left.-1.29) \mathrm{kg} / \mathrm{m}^{2} ; \quad P<0.0001\right)$ and waist circumference (mean difference $-6.29(95 \% \mathrm{CI}-7.85,-4.73) \mathrm{cm} ; P<0.0001)$, as

Table 1. Demographic and health characteristics of participants at baseline

(Number of participants and percentages, $n 54$ )

\begin{tabular}{|c|c|c|}
\hline Variable & $n$ & $\%$ \\
\hline \multicolumn{3}{|l|}{ Sex } \\
\hline Male & 22 & $40 \cdot 7$ \\
\hline Female & 32 & $59 \cdot 3$ \\
\hline \multicolumn{3}{|l|}{ Ethnicity } \\
\hline Caucasian & 52 & $96 \cdot 3$ \\
\hline Pakistani & 1 & 1.9 \\
\hline Other Asian background & 1 & 1.9 \\
\hline \multicolumn{3}{|l|}{ Marital status } \\
\hline Married/live with a partner & 1 & 1.9 \\
\hline Single & 53 & 98.2 \\
\hline \multicolumn{3}{|l|}{ Type of support } \\
\hline Lives independently & 4 & 7.4 \\
\hline Family carer & 17 & 31.5 \\
\hline Paid carer & 33 & $61 \cdot 1$ \\
\hline \multicolumn{3}{|l|}{ Down's syndrome } \\
\hline Yes & 13 & $24 \cdot 1$ \\
\hline \multicolumn{3}{|l|}{ Level of intellectual disabilities } \\
\hline Mild & 17 & 31.5 \\
\hline Moderate & 17 & 31.5 \\
\hline Severe & 19 & $35 \cdot 2$ \\
\hline Profound & 1 & 1.9 \\
\hline \multicolumn{3}{|l|}{ Epilepsy, seizures or fits } \\
\hline Yes & 14 & $25 \cdot 9$ \\
\hline \multicolumn{3}{|c|}{ Registered blind or partially sighted } \\
\hline Yes & 4 & 7.4 \\
\hline \multicolumn{3}{|l|}{ Hearing impairment } \\
\hline Yes & 12 & $22 \cdot 2$ \\
\hline \multicolumn{3}{|l|}{ High blood pressure } \\
\hline Yes & 11 & 20.4 \\
\hline \multicolumn{3}{|l|}{ High cholesterol } \\
\hline Yes & 12 & $22 \cdot 2$ \\
\hline \multicolumn{3}{|l|}{ Type 2 diabetes } \\
\hline Yes & 5 & $9 \cdot 3$ \\
\hline
\end{tabular}


Table 2. Comparison of anthropometric outcome measures at 24 weeks from baseline (analysis set; $n$ 47)

(Mean values, standard deviations and $95 \%$ confidence intervals)

\begin{tabular}{|c|c|c|c|c|c|c|c|}
\hline \multirow[b]{2}{*}{ Outcome } & \multicolumn{2}{|c|}{ Baseline } & \multicolumn{2}{|c|}{24 weeks } & \multirow{2}{*}{$\begin{array}{c}\text { Mean difference } \\
\text { (24 weeks - baseline) }\end{array}$} & \multirow[b]{2}{*}{$95 \% \mathrm{Cl}$} & \multirow[b]{2}{*}{$P^{*}$} \\
\hline & Mean & SD & Mean & SD & & & \\
\hline \multicolumn{8}{|l|}{ Primary outcome } \\
\hline Change in weight at 24 weeks $(\mathrm{kg})$ & $100 \cdot 6$ & $26 \cdot 8$ & $96 \cdot 1$ & $26 \cdot 9$ & -4.47 & $-5.91,-3.03$ & $<0.0001$ \\
\hline \multicolumn{8}{|l|}{ Secondary outcomes } \\
\hline Change in BMI at 24 weeks $\left(\mathrm{kg} / \mathrm{m}^{2}\right)$ & $40 \cdot 0$ & 8.03 & $39 \cdot 2$ & $8 \cdot 2$ & -1.82 & $-2 \cdot 36,-1 \cdot 29$ & $<0.0001$ \\
\hline Change in waist circumference at 24 weeks $(\mathrm{cm})$ & $122 \cdot 1$ & $15 \cdot 7$ & $115 \cdot 8$ & $16 \cdot 7$ & $-6 \cdot 29$ & $-7 \cdot 85,-4 \cdot 73$ & $<0.0001$ \\
\hline
\end{tabular}

* $P$ value obtained from a paired $t$ test

shown in Table 2. Of the forty-seven participants who completed the intervention, seventeen (36.17\%) lost 5\% or more of their initial body weight from baseline, six participants (12.8\%) increased their weight and twenty-four (51\%) participants lost between 0 and $5 \%$ of their initial body weight.

\section{Change in physical activity and sedentary behaviour}

Baseline physical activity data collected with accelerometers were available for forty-five participants. Participants lived sedentary lifestyles, spending a mean of 612 (SD $121.75) \mathrm{min} / \mathrm{d}$ sedentary according to the objective data collected with the GT1M. This equated to 87.9 (SD 7.7) \% of the time spent wearing the accelerometer. The mean time spent in light-intensity physical activity was 69.9 (SD 43.83) $\mathrm{min} / \mathrm{d}$, which was $10 \cdot 1$ (SD $6 \cdot 0$ ) \% of the time spent wearing the accelerometer. Participants spent a mean of $13 \cdot 1$ (SD 16.2) $\mathrm{min} / \mathrm{d}$ in moderate-to-vigorous-intensity physical activity at baseline, which was $2 \cdot 0$ (SD $2 \cdot 7$ ) \% of the total time monitored.

There were baseline and follow-up accelerometer data available for thirty-three participants (Table 3). IPAQ data were available for all forty-seven participants who completed the intervention (Table 4). From the accelerometer data, the percentage of time spent in light-intensity physical activity increased significantly (mean difference 1.87 (95\% CI $0 \cdot 23$, 3.51) $\mathrm{min} ; P=0.027)$. There were also significant post-intervention changes in the absolute (mean difference - 41.40 (95\% CI $-62.45,-20.35) \mathrm{min} ; P=0.00034)$ and percentage of time (mean difference -2.60 (95\% CI $-4.58,-0.62) \%$; $P=0.012$ ) spent in sedentary behaviour. However, none of the IPAQ-S measures changed significantly between baseline and follow-up.

All participants at baseline and follow-up were sedentary for more than $4 \mathrm{~h} / \mathrm{d}$, and no participants met the public health recommendation of a minimum of 150 min of moderate-intensity physical activity/week.

\section{Predictors of weight loss}

None of the variables shown in Table 5 was shown to be significant predictors of weight loss. Similarly, there were no significant between-group differences in weight loss in $\mathrm{kg}$ in Table 6. However, the results for Down's syndrome in Table 5 and the level of intellectual disabilities, shown in Tables 5 and 6, approach significance. This suggests that individuals participating in the TAKE 5 intervention with Down's syndrome or mild/moderate levels of intellectual disabilities may be more likely to achieve weight loss.

Table 3. Comparison of physical activity outcome measures at 24 weeks from baseline (analysis set - accelerometer; $n$ 33)

(Mean values, standard deviations and $95 \%$ confidence intervals)

\begin{tabular}{|c|c|c|c|c|c|c|c|c|}
\hline \multirow[b]{2}{*}{ Outcome } & \multicolumn{2}{|c|}{ Baseline } & \multicolumn{2}{|c|}{24 weeks } & \multirow{2}{*}{$\begin{array}{c}\text { Mean difference } \\
(24 \text { weeks - baseline) }\end{array}$} & \multirow[b]{2}{*}{$95 \% \mathrm{Cl}$} & \multirow[b]{2}{*}{$P^{\star}$} & \multirow[b]{2}{*}{$P \dagger$} \\
\hline & Mean & SD & Mean & SD & & & & \\
\hline \multicolumn{9}{|l|}{ Secondary outcomes } \\
\hline $\begin{array}{l}\text { Mean change in light-intensity } \\
\text { physical activity/d at } 24 \text { weeks (min) }\end{array}$ & $73 \cdot 4$ & $46 \cdot 8$ & $81 \cdot 3$ & $45 \cdot 6$ & $7 \cdot 90$ & $-5 \cdot 29,21 \cdot 09$ & 0.23 & 0.13 \\
\hline $\begin{array}{l}\text { Mean change in moderate-to- } \\
\text { vigorous-intensity } \\
\text { physical activity/d at } 24 \text { weeks (min) }\end{array}$ & $14 \cdot 2$ & $17 \cdot 5$ & $17 \cdot 8$ & $17 \cdot 3$ & 3.61 & $-0.70,7.93$ & 0.098 & 0.12 \\
\hline $\begin{array}{l}\text { Mean change in sedentary behaviour/d } \\
\text { at } 24 \text { weeks (min) }\end{array}$ & $623 \cdot 3$ & $121 \cdot 5$ & $581 \cdot 9$ & $116 \cdot 4$ & -41.40 & $-62 \cdot 45,-20 \cdot 35$ & 0.00034 & 0.00018 \\
\hline $\begin{array}{l}\text { Mean change in percentage of time spent } \\
\text { in light-intensity physical activity (min) }\end{array}$ & $10 \cdot 4$ & $6 \cdot 2$ & $12 \cdot 3$ & $6 \cdot 0$ & 1.87 & $0.23,3.51$ & 0.027 & 0.019 \\
\hline $\begin{array}{l}\text { Mean change in percentage of time spent } \\
\text { in moderate-to-vigorous-intensity } \\
\text { physical activity (min) }\end{array}$ & $2 \cdot 1$ & $3 \cdot 1$ & $2 \cdot 7$ & 3.0 & -0.57 & $-0.05,1 \cdot 19$ & 0.072 & 0.092 \\
\hline $\begin{array}{l}\text { Mean change in percentage of time spent } \\
\text { in sedentary behaviour (min) }\end{array}$ & $87 \cdot 5$ & $8 \cdot 0$ & 84.9 & $8 \cdot 0$ & $-2 \cdot 60$ & $-4.58,-0.62$ & 0.012 & 0.0086 \\
\hline
\end{tabular}

* $P$ value was obtained from a paired $t$ test.

$\dagger P$ value was obtained from Wilcoxon's signed-rank test. 
Table 4. Comparison of physical activity outcome measures at 24 weeks from baseline (analysis set - questionnaire; $n$ 47)

(Mean values, standard deviations and $95 \%$ confidence intervals)

\begin{tabular}{|c|c|c|c|c|c|c|c|c|}
\hline \multirow[b]{2}{*}{ Outcome } & \multicolumn{2}{|c|}{ Baseline } & \multicolumn{2}{|c|}{24 weeks } & \multirow{2}{*}{$\begin{array}{c}\text { Mean difference } \\
\text { (24 weeks - baseline) }\end{array}$} & \multirow[b]{2}{*}{$95 \% \mathrm{Cl}$} & \multirow[b]{2}{*}{$P^{*}$} & \multirow[b]{2}{*}{$P \dagger$} \\
\hline & Mean & SD & Mean & SD & & & & \\
\hline \multicolumn{9}{|l|}{ Secondary outcomes } \\
\hline $\begin{array}{l}\text { Change in moderate-to-vigorous-intensity } \\
\text { physical activity in previous } 7 \mathrm{~d} \text { at } \\
24 \text { weeks (min) }\end{array}$ & $55 \cdot 4$ & $89 \cdot 0$ & $81 \cdot 6$ & $102 \cdot 4$ & $26 \cdot 24$ & $-2 \cdot 26,54 \cdot 74$ & 0.070 & 0.104 \\
\hline $\begin{array}{l}\text { Change in time walking in previous } 7 \mathrm{~d} \\
\text { at } 24 \text { weeks }(\mathrm{min})\end{array}$ & $48 \cdot 7$ & $54 \cdot 3$ & $63 \cdot 5$ & $55 \cdot 0$ & $14 \cdot 72$ & $-8 \cdot 10,37 \cdot 55$ & 0.20 & 0.058 \\
\hline Change in time sitting/d at 24 weeks (min) & 557.4 & 189.4 & 531.4 & 173.6 & -25.98 & $-71.29,19.34$ & 0.25 & 0.22 \\
\hline
\end{tabular}

${ }^{*} P$ value was obtained from a paired $t$ test.

$\dagger P$ value was obtained from Wilcoxon's signed-rank test.

There was no significant difference in weight loss for participants living with family or paid carer support.

\section{Discussion}

This is the first study to examine the effectiveness of a multicomponent weight-loss intervention, incorporating an energydeficit diet, for adults with intellectual disabilities and obesity. The significant change in body weight and waist circumference suggests that TAKE 5 may be an effective weight-loss intervention for adults with intellectual disabilities and obesity. Since there was a low dropout rate, a multi-component weight-loss intervention that includes an energy-deficit diet appears acceptable to adults with intellectual disabilities and obesity.

Although the changes in moderate-to-vigorous levels of physical activity were not significant, participants made clinically relevant changes to their levels of sedentary behaviour and light-intensity physical activity. There is evidence that sedentary behaviour is a significant risk factor for chronic disease, independent of the levels of moderate-to-vigorousintensity physical activity ${ }^{(31)}$. Therefore, the finding that TAKE 5 successfully supported participants to make initial positive changes in sedentary behaviour and light-intensity physical activity is important, and if maintained, it would be associated with health improvement. It is unclear whether the changes in the participants' physical activity and sedentary behaviour were attributable to increased participation in housework, walking or sport and exercise. For some individuals, even though carers were motivated to provide support to increase activity levels, the level of paid carer support available acted as a barrier to increased activity outside the home environment. Barriers to change will be examined more fully in an accompanying qualitative paper, but a relevant example was where a support package did not provide time for one-to-one support that could be used to visit a local leisure centre, or go for a walk. Hence, it was important to identify opportunities for physical activity such as housework or gardening. The finding that the intervention led to positive changes in light-intensity physical activity and sedentary behaviour suggests that participants did increase their levels of physical activity in the home environment.

While the between-group differences in weight loss were not statistically significant, there was a trend towards participants with Down's syndrome, or higher ability levels being more likely to lose $5 \%$ or more of their body weight. It is not clear from the study why this was the case. The findings suggest that there is no need for a specific intervention for individuals with Down's syndrome. Further work should be done to clarify whether weight-loss interventions are more

Table 5. Association between participants achieving a weight loss of $5 \%$ or more of their initial body weight and patient characteristics (analysis set; $n 47)^{*}$

(Odds ratios and $95 \%$ confidence intervals)

\begin{tabular}{llll}
\hline Variable & OR $\dagger$ & $95 \% \mathrm{Cl}$ & $P$ \\
\hline Age (1 year) & 0.97 & $0.92,1.02$ & 0.19 \\
Sex (male v. female) & 1.05 & $0.31,3.52$ & 0.94 \\
Family $v$. paid carer & 1.33 & $0.57,3.13$ & 0.67 \\
Down's syndrome & 3.50 & $0.90,13.66$ & 0.071 \\
Intellectual disabilities mild/moderate $v$. severe/profound & 4.08 & $0.97,17.21$ & 0.055 \\
Epilepsy seizures or fits & 0.85 & $0.21,3.37$ & 0.81 \\
Registered blind or partially sighted & 1.87 & $0.24,14.61$ & 0.55 \\
Hearing impairment/ear problems & 1.67 & $0.42,6.59$ & 0.47 \\
Type 2 diabetes & 0.56 & $0.05,5.88$ & 0.63 \\
Problems in walking because of weight & 0.40 & $0.11,1.53$ & 0.18 \\
Prescribed obesogenic medication & 0.86 & $0.18,3.98$ & 0.84 \\
\hline
\end{tabular}

* Analysis was performed using univariate logistic regression.

† Odds ratios and confidence intervals are for increases of units specified by $95 \% \mathrm{Cl}$ values for continuous measures, i.e. for age increase of 1 year. Odds ratios and confidence intervals are for yes $v$. no for categorical variables, unless otherwise stated. 
Table 6. Comparison of weight change $(\mathrm{kg})$ at 24 weeks from baseline and patient characteristics (analysis set; $n$ 47)

(Mean values and $95 \%$ confidence intervals)

\begin{tabular}{|c|c|c|c|c|c|}
\hline Variable & $\begin{array}{c}\text { Group } 1 \\
\text { Mean }\end{array}$ & $\begin{array}{l}\text { Group } 2 \\
\text { Mean }\end{array}$ & $\begin{array}{l}\text { Mean difference in weight } \\
\text { change at } 24 \text { weeks }\end{array}$ & $95 \% \mathrm{Cl}$ & $P$ \\
\hline Age (group 1: $\leq 49$ years; group $2:>49$ years) & -5.22 & -3.81 & -1.40 & $-4.29,1.48$ & 0.33 \\
\hline Sex (group 1: male; group 2: female) & -5.39 & -3.85 & -1.55 & $-4.47,1.38$ & 0.29 \\
\hline Type of support (group 1: family carer; group 2: paid carer) & -5.07 & -4.24 & -0.83 & $-4.07,2.41$ & 0.61 \\
\hline Down's syndrome & $-5 \cdot 53$ & $-4 \cdot 11$ & -1.43 & $-4.73,1.88$ & 0.39 \\
\hline Intellectual disability (group 1: mild/moderate; group 2: severe/profound) & -5.46 & $-2 \cdot 72$ & -2.74 & $-5 \cdot 66,0.17$ & 0.064 \\
\hline Epilepsy seizures or fits & -4.07 & -4.61 & 0.54 & $-2.79,3.88$ & 0.74 \\
\hline Registered blind or partially sighted & $-7 \cdot 11$ & -4.23 & $-2 \cdot 89$ & $-8.03,2.25$ & 0.26 \\
\hline Hearing impairment/ear problems & -4.25 & -4.54 & 0.29 & $-3.14,3.73$ & 0.86 \\
\hline Type 2 diabetes & -1.81 & -4.72 & $2 \cdot 91$ & $-2 \cdot 23,8.05$ & 0.26 \\
\hline Problems in walking because of weight & -4.67 & -4.36 & -0.31 & $-3 \cdot 34,2 \cdot 71$ & 0.84 \\
\hline Prescribed obesogenic medication & -4.41 & -4.49 & 0.07 & $-3.62,3.77$ & 0.97 \\
\hline
\end{tabular}

likely to be successful for more-able individuals. One possible reason is that more-able individuals have baseline activity levels that match the PAL of 1.4 used in the present study, while less-able participants are less active and require a lower PAL. Alternatively, more-able participants may find it easier to contribute to goal setting and self-monitoring, thus maintaining their motivation for behavioural change. These possibilities, factors related to the involvement of carers, and the differential effects of barriers to changing the diet and PAL should be examined in future studies.

There was no significant difference in weight loss between participants living with family and paid carers. Although the numbers in the two groups are small, this result is encouraging, as it suggests that TAKE 5 may be equally effective for participants receiving different types of support.

\section{Comparison with previous studies}

Clinical guidelines vary in whether they recommend different $^{(6,7)}$ or similar ${ }^{(8)}$ interventions for individuals with a BMI in the overweight and obesity categories. TAKE 5 was designed for use with adults with intellectual disabilities who want to lose weight, regardless of whether they are overweight or obese. However, in keeping with the guidelines available at the time of the start of the study ${ }^{(6,7)}$ and clinical priorities, this study included only individuals with obesity.

Most studies reporting weight loss in adults with intellectual disabilities have used samples that included individuals with a BMI in the overweight and obesity categories ${ }^{(17,18,32-34)}$. Several studies have also included participants of normal weight ${ }^{(35-37)}$. Comparison with the results of these previous studies is difficult because they have not reported the results separately for participants with obesity. Furthermore, none of these studies reports the percentage of participants achieving a clinically relevant weight loss of $5 \%$ of initial body weight.

Only one weight-loss study reporting comparable results for adults with intellectual disabilities and obesity has been published previously ${ }^{(38)}$. This study examined the effectiveness of a 10-week behavioural, self-control intervention ${ }^{(17)}$ in six adults with mild intellectual disabilities. Although guidance on healthy eating was included, this intervention does not include an energy-deficit diet. At baseline, all participants had a BMI over 30 (mean BMI 44.3 ) $\mathrm{kg} / \mathrm{m}^{2}$, and the mean weight of the six participants was $114.7 \mathrm{~kg}$. At the end of the 10 -week intervention, mean weight loss was $2.5 \mathrm{~kg}$. Of the six participants, one lost $5.6 \%$ of their initial body weight, three lost between 1.9 and $2.6 \%$, one participant stayed at the same weight and one gained $0.3 \%$ of initial weight. It is clearly not possible to reach conclusions on the effectiveness of the intervention from such a small study. In previous studies using the same intervention with adults with intellectual disabilities who were overweight and obese, the intervention appeared to be associated with significant weight $\operatorname{loss}^{(17,18)}$. However, insufficient data were provided to consider the effectiveness for adults with obesity independently.

Since no data from studies using an energy-deficit diet with participants with intellectual disabilities were available, the results are compared with the findings of a meta-analysis of five studies that did not include participants with intellectual disabilities $^{(7)}$. The mean weight loss reported in these five studies was -4.60 (range $-3.33,-5.87$ ) $\mathrm{kg}$, compared with $-4.47(95 \%$ CI $-5.91,-3.03) \mathrm{kg}$ for TAKE 5. Although the different samples and designs make direct comparison difficult, the significant changes in weight and waist circumference suggest that TAKE 5 may be an effective weight-loss intervention for adults with intellectual disabilities and obesity.

\section{Strengths and limitation}

This is the first study reporting the effectiveness and acceptability of a weight-loss intervention incorporating an energydeficit diet for adults with intellectual disabilities and obesity. Furthermore, the use of a multi-component weight-loss intervention is in keeping with current recommendations from clinical guidelines ${ }^{(6-8)}$. Although these recommendations are derived from general population research, the results suggest that combining these three components is effective in supporting weight loss in adults with intellectual disabilities and obesity. To ensure the accessibility of the intervention and the utility across a range of abilities, TAKE 5 is designed to be delivered on a one-to-one basis, with the involvement of carers where appropriate. The cost implications of this 
should be examined in future controlled studies. If future studies confirm the efficacy of TAKE 5, consideration could be given to adapting the methods to a group setting. As well as reducing costs, this could potentially make use of the potential benefits described in group studies ${ }^{(39)}$ involving participants who do not have intellectual disabilities.

In keeping with the evidence from previous studies, TAKE 5 was designed to incorporate support from carers where possible ${ }^{(17,18)}$. This ensures that the intervention is accessible to individuals regardless of their ability level and maximises the opportunity for knowledge and skill development to support behavioural change. Behavioural methods to maintain carer motivation were incorporated into the intervention, and specific information to improve carer knowledge of the principles of healthy eating and PAL was provided ${ }^{(40)}$. Carers were encouraged to involve participants in decisions about meals, shopping for food and cooking, where possible. Similarly, as a means to increasing physical activity, carers were encouraged to involve participants in household tasks. However, the degree of participant involvement in decisions about dietary changes and physical activity varied depending on an individual participant's level of abilities.

No formal method to measure participant or carer adherence was incorporated into the study design. Adherence to the intervention varied between participants. However, the intervention aimed to allow identification of problems specific to an individual and adoption of a collaborative approach to improve adherence between the participant, carers and the health professional. As part of the intervention, selfmonitoring of diet and physical activity between sessions using diaries and monitoring of weight change provided relevant information on adherence. The health professionals made use of this information to discuss what was going well and also barriers to change, particularly in relation to an individual participant's goals. Where barriers were identified, possible solutions were discussed and incorporated into subsequent goal setting. There are no formal data to examine whether there was a significant difference in participants' adherence to agreed changes in diet and physical activity. However, the significant changes in outcome measures relevant to weight and physical activity suggest that participants made changes in both these lifestyle areas. In future studies, consideration will be given to incorporating more formal methods to examine adherence to the intervention and the achievement of goals between sessions.

Data collection was carried out by a research assistant who was not involved in the delivery of the intervention, following explicit methods for anthropometric measures. The same research assistant was involved in pre- and post-intervention measurements and therefore was not blind to which measurement was being made. In addition to change in weight, change in measured levels of physical activity and sedentary behaviour allowed consideration of the effectiveness of the different components of TAKE 5. The use of GT1M accelerometers to collect physical activity data gave an objective measure. Given the complex nature of the sample population, it was expected that some participants would not be able to make use of the accelerometers. However, full accelerometer data were available for over $70 \%$ of individuals completing the intervention. Nonetheless, to take account of incomplete accelerometer data, the use of a questionnaire as a second measure of physical activity should be considered in future studies involving participants with intellectual disabilities.

To calculate the total energy expenditure of participants, a PAL factor of 1.4 was used in the present study. The use of accelerometers provides objective evidence that, given the high baseline levels of the sedentary behaviour of participants, it could be that a PAL of 1.4 overestimates the total energy expenditure of participants. Future studies should consider using a lower $\mathrm{PAL}^{(41)}$.

A longer follow-up than the 24-week period in the present study is important to examine of weight-loss maintenance. However, the 24-week follow-up from baseline allowed preliminary examination of the effectiveness and acceptability of the weight-loss component of TAKE 5.

An important limitation in the study methodology is the absence of a control group. An open pilot study was preferred on the basis that there was very little evidence on the effectiveness and acceptability of weight-loss interventions for adults with intellectual disabilities and obesity. The present open study allows the intervention to be revised and provides data relevant to calculating the necessary sample size, before a larger, controlled study.

\section{Clinical implications}

There is available literature describing the clinical interventions currently offered to adults with intellectual disabilities and obesity. Anecdotally, generic weight-loss services are reluctant to offer interventions on the basis that there is very little evidence on the effectiveness and acceptability of weight-loss interventions for this specific group of individuals. The priority for specialist intellectual disability services has most often been individuals with profound and complex needs who are underweight. Therefore, it may be the case in many areas that adults with intellectual disabilities and obesity are not offered support with weight loss by statutory services. While there is a clear need to develop the evidence base further, the present study provides support for the use of multi-component weight-loss interventions that incorporate energy-deficit diets, as recommended by clinical guidelines. Partnership working between generic weight loss and specialist intellectual disability services is one model that can support the provision of accessible services.

\section{Conclusions}

The results suggest that TAKE 5 is an effective weight-loss intervention for adults with intellectual disabilities and obesity. An energy-deficit diet within a multi-component intervention was acceptable to participants with intellectual disabilities. Given the limited evidence base, there is a need for future studies to examine the effectiveness of multi-component weight-loss interventions in controlled studies. 


\section{Acknowledgements}

We would like to thank the study participants and family and paid carers. We also thank Kelly Hay and Kate Livingstone who provided administrative support for the study. The research was funded by the Chief Scientist Office, Scottish Government Health Department (reference CZG/2/362). C. A. M. was the principal investigator, and was responsible for the conduct of the study and the final content of the manuscript. C. R. H., S. B., H. M. and V. P. were involved in the design and the management of the study. C. A. M., C. R. H., S. B., S. M., N. R., C. P., S. Mac., L. M. and D. S. were responsible for the development of the intervention. S. Mac., V. P. and H. M. were responsible for the data collection and management. All authors contributed to writing the drafts of the manuscript and approved the final version. None of the authors has any conflict of interests to declare.

\section{References}

1. World Health Organization (2004) Obesity: Preventing and Managing the Global Epidemic. Geneva: World Health Organization.

2. Haslam DW \& James WP (2005) Obesity. Lancet 366, $1197-1209$

3. Guh DP, Zhang W, Bansback N, et al. (2009) The incidence of co-morbidities related to obesity and overweight: a systematic review and meta-analysis. BMC Public Health 9, 88.

4. Whitlock G, Lewington S, Sherliker P, et al. (2009) Bodymass index and cause-specific mortality in 900000 adults: collaborative analyses of 57 prospective studies. Lancet 373, 1083-1096.

5. Avenell A, Broom J, Brown TJ, et al. (2004) Systematic review of the long-term effects and economic consequences of treatments for obesity and implications for health improvement. Health Technol Assess 8, 1-182.

6. National Health and Medical Research Council (2003) Clinical Practice Guidelines for the Management of Overweight and Obesity in Adults. Canberra: National Health and Medical Research Council.

7. National Institute for Health and Clinical Excellence (2006) Obesity: the Prevention, Identification, Assessment and Management of Overweight and Obesity in Adults and Children. London: National Institute for Health and Clinical Excellence.

8. Scottish Intercollegiate Guideline Network (2010) Management of Obesity: A National Clinical Guideline. Edinburgh: Scottish Intercollegiate Guideline Network.

9. Robertson J, Emerson E, Gregory N, et al. (2000) Lifestyle related risk factors for poor health in residential settings for people with intellectual disabilities. Res Dev Disabil 21, 469-486.

10. Hove O (2004) Weight survey on adult persons with mental retardation living in the community. Res Dev Disabil 25, 9-17.

11. Yamaki K (2005) Body weight status among adults with intellectual disability in the community. Ment Retard 43, 1-10.

12. Melville CA, Cooper S-A, Morrison J, et al. (2008) The prevalence and determinants of obesity in adults with intellectual disabilities. J Appl Res Intellect Disabil 21, 425-437.

13. Bhaumik S, Watson JM, Thorp CF, et al. (2008) Body mass index in adults with intellectual disability: distribution, associations and service implications: a population-based prevalence study. J Intellect Disabil Res 52, 287-298.
14. Emerson E (2005) Underweight, obesity and exercise among adults with intellectual disabilities in supported accommodation in Northern England. J Intellect Disabil Res 49, $134-143$

15. Hamilton S, Hankey CA, Miller S, et al. (2007) A review of weight loss interventions for adults with intellectual disabilities. Obes Rev 8, 339-345.

16. Scottish Executive (2000) Adults with Incapacity (Scotland) Act. Edinburgh: The Stationery Office.

17. Fox RA, Haniotes H \& Rotatori A (1984) A streamlined weight loss program for moderately retarded adults in a sheltered workshop setting. Appl Res Ment Retard 5, 69-79.

18. Fox RA, Rosenberg R \& Rotatori AF (1985) Parent involvement in a treatment program for obese retarded adults. J Behav Ther Exp Psychiatry 16, 45-48.

19. Food Standards Agency (2009) The Eatwell Plate. London: Food Standards Agency. www.eatwelll.gov.uk (accessed on 10 June 2010).

20. Schofield WN, Schofield C \& James WPT (1985) Basal metabolic rate - review and prediction, together with annotated bibliography of source material. Hum Nutr Appl Nutr 39C, 5-96.

21. Committee on the Medical Aspects of Food Policy (1991) Dietary Reference Values for Food Energy and Nutrients for the United Kingdom. London: HMSO.

22. NHS Health Scotland (2009) Five Year Review of "Let's Make Scotland More Active" - A Strategy for Physical Activity. Edinburgh: NHS Health Scotland.

23. Leslie WS, Hankey CR \& Lean ME (2007) Weight gain as an adverse effect of some commonly prescribed drugs: a systematic review. QJM 100, 395-404.

24. Cooper SA (1997) Epidemiology of psychiatric disorders in elderly compared with younger adults with learning disabilities. Br J Psychiatry 170, 375-380.

25. Sparrow SS, Balla DA \& Cicchetti DV (1984) Obtaining raw scores and derived scores. In A Revision of the Vinelands Social Maturity Scale, pp. 89-109 [E. A. Doll, editor]. Minneapolis, MN: American Guidance Service, Inc.

26. World Health Organization Expert Committee (1995) Physical Status: The Use and Interpretation of Anthropometry. Geneva: World Health Organization.

27. Lean ME, Han TS \& Morrison CE (1995) Waist circumference as a measure for indicating need for weight management. BMJ 311, 158-161.

28. Penpraze V, Reilly JJ, MacLean C, et al. (2006) Monitoring of physical activity in young children: how much is enough? Pediatr Exerc Sci 18, 483-491.

29. Freedson PS, Melanson E \& Sirard J (1998) Calibration of the Computer Science and Applications Inc. accelerometer. Med Sci Sports Exerc 30, 777-781.

30. Craig CL, Marshall AL, Sjostrom M, et al. (2003) International physical activity questionnaire: 12-country reliability and validity. Med Sci Sports Exerc 35, 1381-1395.

31. Ekblom-Bak E, Hellenius ML \& Ekblom B (2010) Are we facing a new paradigm of inactivity physiology? Br J Sports Med 44, 587-588.

32. McCarran MS \& Andrasik F (1990) Behavioral weight-loss for multiply-handicapped adults: assessing caretaker involvement and measures of behavior change. Addict Behav 15, $13-20$.

33. Ewing G, McDermott S, Thomas-Koger M, et al. (2004) Evaluation of a cardiovascular health program for participants with mental retardation and normal learners. Health Educ Behav 31, 77-87. 
34. Zoppo A \& Asteria C (2008) Obesity treatment and cardiovascular prevention in mentally retarded subjects. Int $J$ Obes 32, 1034.

35. Marshall D, McConkey R \& Moore G (2003) Obesity in people with intellectual disabilities: the impact of nurse-led health screenings and health promotion activities. $J$ Adv Nurs 41, 147-153.

36. Chapman MJ, Craven MJ \& Chadwick DD (2005) Fighting fit? An evaluation of health practitioner input to improve healthy living and reduce obesity for adults with learning disabilities. J Intellect Disabil 9, 131-144.

37. Wu CL, Lin JD, Hu J, et al. (2010) The effectiveness of healthy physical fitness programs on people with intellectual disabilities living in a disability institution: six-month short-term effect. Res Dev Disabil 31, 713-717.
38. Sailer AB, Miltenberger RG, Johnson B, et al. (2006) Evaluation of a weight loss treatment program for individuals with mild mental retardation. Child Fam Behav Ther 28, $15-28$.

39. Paul-Ebhohimhen V \& Avenell A (2009) A systematic review of the effectiveness of group versus individual treatments for adult obesity. Obes Facts 2, 17-24.

40. Melville CA, Hamilton S, Miller S, et al. (2009) Carer knowledge and perceptions of healthy lifestyles for adults with intellectual disabilities. I Appl Res Intellect Disabil 22, 298-306.

41. Leslie WS, Lean ME, Baillie HM, et al. (2002) Weight management: a comparison of existing dietary approaches in a work-site setting. Int J Obes 26, 1469-1475. 